\title{
Advantages of Petri-Net Modeling and Simulation for Biological Networks
}

\author{
Ralf Hofestädt ${ }^{*}$ \\ Bielefeld University, AG Bioinformatics, Universitätsstr. 25, 33615 Bielefeld, Germany. \\ * Corresponding author. Tel.: +49521 1065263; email: ralf.hofestaedt@uni-bielefeld.de. \\ Manuscript submitted January 30, 2017; accepted March 25, 2017. \\ doi: 10.17706/ijbbb.2017.7.4.221-229
}

\begin{abstract}
During the last 20 years Petri-Nets have attracted more and more attention to help to model and simulate biological networks. To model the identified features of cellular processing it was necessary to extend the basic definition of Petri-Nets. Regarding the published papers, it seems clear that hybrid functional Petri-Nets are the adequate method to model complex biological networks today. Furthermore, different higher Petri-Nets are available to realize the adequate modeling of cellular processes. This paper will present the advantages of Petri-Nets for modeling and simulation of biological networks.
\end{abstract}

Key words: Biological networks, modeling, Petri-Nets, simulation.

\section{Introduction}

Modeling and simulation of metabolic networks is a part of Bioinformatics and Chemical Informatics. However, first publications can be found published in the field of Biophysics/Biomathematics more than 50 years ago. Comparing these papers we can identify two classes of models and simulation tools. Discrete models like automata or formal languages and analytical models like differential equations. The Kauffman network approach [1] was one of the first discrete models for gene controlled networks. At the end of the last century more and more discrete models were published for modeling and simulation of metabolic networks. The reason to use discrete models was/is the gap of relevant quantitative molecular data and knowledge. Behind automata, formal languages and graphs the Petri-Net approach became popular for modeling of these processes at the end of the last century [2], [3]. During the last years the situation of quantitative molecular data changed rapidly based on new cellular analysis technologies. Therefore, "Quantitative Biology" became a new research topic of molecular Biology. Until now it is still an open question, which formal system will be of practical use for modeling and simulation of cellular processes. This paper will focus to the Petri-Net approach and will present their key features. Furthermore, the advantages of Petri-Nets for modeling and simulation of biological networks will be discussed.

\section{Related Works}

During the last decades different methods and tools for the simulation and modeling of networks were developed and published. In this paper we focus only to the most relevant systems and identify their key features. The most relevant system for modeling and simulation of biochemical networks is Gepasi/Copasi [4], which is perfect to model kinetic aspects and biochemical reactions. The idea of the E-Cell [5] is to model and simulate a gene controlled virtual biochemical organism. The Cellillustrator [6] allows the 
qualitative and quantitative simulation of biological networks. The first object oriented system was Dbsolve [7], which is useful for modeling, analysis, and optimization of biological networks. Virtual Cell [8] is a modular computational framework that permits construction of models, application of numerical solvers to perform simulations, and analysis of simulation results. Cell Designer [9] is a modeling tool of biochemical networks with a graphical user interface. Networks are drawn based on the process diagram, with graphical notation system proposed by Kitano [10]. Several Petri-Net tools have been developed. In this chapter we focus to the main tools which are already used for modeling and simulation of biological networks. The Visual Object Net ++ (VON++) is a Petri-Net CAE tool that supports mixed continuous and discrete event Petri-Nets [11]. The system was used to model gene regulatory processes and metabolic pathways. Snoopy is a software tool to design and animate hierarchical graphs, among other Petri-Nets [12]. It is in use for the verification of technical systems, especially software-based systems, as well as for the validation of natural systems, i.e. biochemical networks as metabolic, signal transduction, gene regulatory networks. THORN is a general-purpose, graphical, discrete-event simulation tool based on a special class of high-level Petri-Nets called Timed Hierarchical Object-Related Nets (THORN). THORN allows the specification of individual tokens, which provide delay times and firing durations for transitions. Furthermore, THORN models can be hierarchically structured [13]. An object-oriented Petri-Net simulation tool for hybrid biological processes for MODELICA applications is presented in [14]. Genomic Object Net (GON) is an environment for simulating and representing biological systems. GON has been further developed into the new tool Cellillustrator [15].

\section{Petri-Net Representation of Biological Networks}

\subsection{Basics}

A Petri-Net $\mathrm{PN}=\left(\mathrm{P}, \mathrm{T}, \mathrm{F}, \mathrm{W}, \mathrm{m}_{0}\right)$ consists of a finite set of transitions $(\mathrm{T})$ and a finite set of places (P), which are connected by directed $\operatorname{arcs}(\mathrm{F}) . \mathrm{F} \subseteq(\mathrm{P} \times \mathrm{T}) \cup(\mathrm{T} \times \mathrm{P})$ is a finite set of arcs. The triple $(\mathrm{P}, \mathrm{T}, \mathrm{F})$ is called net and $\mathrm{W}$ is the weight function (W: $\mathrm{F} \rightarrow \mathrm{IN}$ ) of the Petri-Net. Regarding the graphical representation (see Fig. 1) places are drawn as circles, transitions are drawn as rectangles and arcs are drawn as directed arrows. Places and transitions are labeled with specific names [2], [16].

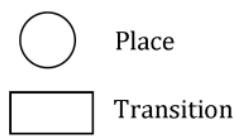

Fig. 1. Place and transition as nodes of the Petri-Net.

Places may contain tokens, which are drawn as dots (see Fig. 2). Tokens can be assigned to places, which is called configuration and $\mathrm{m}_{0}$ denotes the start configuration of the Petri-Net.

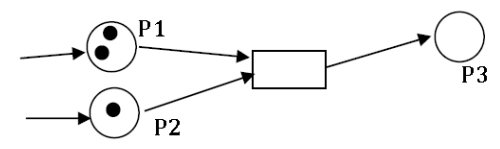

Fig. 2. Petri-Net representing tokens with configuration $(2,1,0)$.

The complete definition of the ordinary Petri-Net is given in [2], which also presents the first paper using Petri-Nets for the modeling of qualitative biochemical reactions. At this point we would like to focus only to the basic definitions and features. A transition is able to fire, if all pre-places represent more or equal tokens than the input arrow announced. If no integer number is placed at the arrow this is interpreted as 1 (see Fig. 
2). If any transition is able to fire this transition has concession. Regarding Fig. 2 the transition has concession and is able to fire. The standard definition of Petri-Nets is saying nothing about the time. Therefore, a transition under concession will or can fire anytime. The firing process will reduce the number of tokens from the pre-places (as much as announced at each arrow) and produce tokens into the post-places (as much as announced at each arrow). In our example the result of the firing process will be shown by Fig. 3.

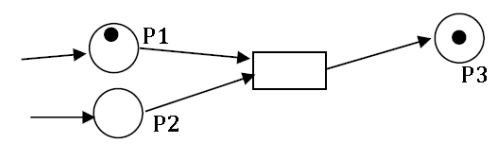

Fig. 3. The result of the firing process regarding Fig. 2 . The new configuration is $(1,0,1)$.

\subsection{Petri-Net Extensions for Modeling of Biological Networks}

The key message of the paper of Reddy et al. [2] was to demonstrate that Petri-Nets are useful for the representation of biochemical reactions and metabolic pathways as demonstrated in Fig. 4.

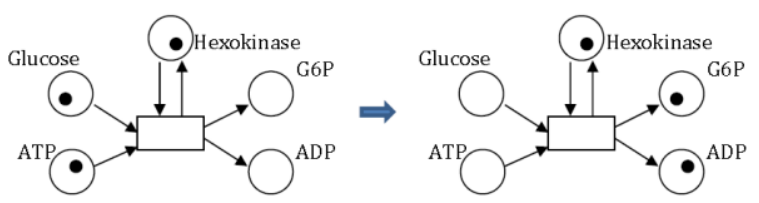

Fig. 4. Petri-Net representation of a enzyme-catalyzed process (glucose into glucose-6-phosphate).

However, the disadvantage of this kind of application presented by Reddy et al. was the modeling of kinetic effects. The work from Hofestädt and Thelen [3] introduced functional Petri-Nets to close this gap. Furthermore, this paper showed that a Petri-Net can also model gene-controlled metabolic processes and cell communication processes. The motivation to define the functional Petri-Net was to allow the quantitative modeling of biological networks. Based on methods of biotechnology more and more quantitative molecular data is available via the internet today. This molecular data allows complex simulations of biological networks. Finally, to allow using real numbers to simulate concentration values was one important aspect to define the Hybrid Functional Petri-Net (HFPN) [17]. The HFPN is an extension of the Hybrid Petri-Net (HPN) [18]. The idea of the HPN was the representation of two kinds of places and transitions allowing calculating discrete and analytical molecular values. Therefore, discrete places (discrete transitions) and the continuous places (continuous transitions) were defined (see Fig. 5). The idea of the continuous place is that nonnegative real numbers can be used, which can be interpreted as the concentration rates of metabolites. Furthermore, two new classes of arcs were defined. Test arcs will not consume tokens and inhibitory arcs can simulate the reaction of biochemical inhibitor molecules.

Drath introduced the Hybrid dynamic net (HDN) [19], which has a similar structure as the HPN. The main difference of HDN and HPN is the definition of the firing process. Based on the HDN and HPN formalisms, Doi et al. [20] introduced the HFPN, which includes both of these features of HPN and HDN. Furthermore, HFPN has the feature of the functional Petri-Nets and allows assigning a function of values to any arc. Based on these ideas several simulation tools were created and implemented. The implementation of this formal description leads to the Cellillustrator simulation shell (http://www.cellillustrator.com/), which allows the modeling of complex biochemical networks including the simulation of concentrations regarding time relevant aspects. Regarding the theory of Petri-Nets we can identify numerous higher Petri-Nets representing different aspects and features. Stochastic Petri-Nets are useful in case of probability aspects 
[21]. Fuzzy Petri-Nets [22], learning Petri-Nets [23] and object oriented Petri-Nets [7] represent only a snapshot of this wide collection of higher Petri-Nets.

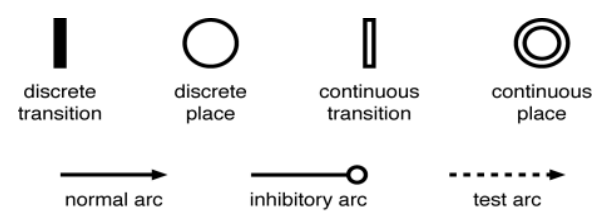

Fig. 5. This figure shows the fundamental symbols of HPN defined in [18].

\section{Petri-Net Extension for the Analysis of Biochemical Features}

\subsection{Biochemical Features}

Today biotechnology methods generate quantitative data based on high throughput machines like DNA sequencing and OMICS analysis. Based on this data for example kinetic biochemical effects can be analyzed and understood. Therefore, models must be able to represent quantitative data (quantitative Biology). The classical method for this kind of biochemical modeling is the definition of differential equations which simulate the molecular behavior. The step from qualitative to quantitative Biology can be realized using quantitative Petri-Nets like Hybrid Petri-Nets. Therefore, special places and transitions are included into this Petri-Net extension (see Fig. 5). Furthermore, this extension allows the modeling of kinetic processes by adding functions to the arcs, which modifies the firing process. Fig. 6 shows a simple Petri-Net representation of a gene controlled biochemical reaction model. Furthermore, we have access to kinetic data directly via database systems like BRENDA (http://www.brenda-enzymes.org/).

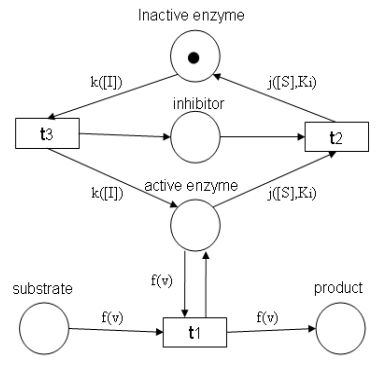

Fig. 6. A functional Petri-Net models the competitive inhibition of an enzymatic controlled biochemical process. Where $\mathrm{V}:=$ reaction rate, $[\mathrm{S}]:=$ substrate concentration, $[\mathrm{P}]:=$ product concentration, $[\mathrm{I}]:=$ inhibitor concentration, $K_{i}:=$ dissociation constant of the inhibitor. $\mathrm{k}, \mathrm{j}$ and $\mathrm{f}$ represent specific functions.

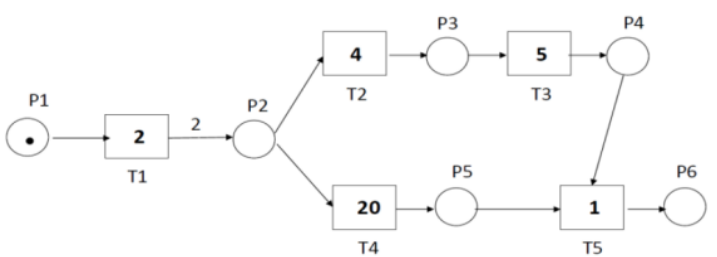

Fig. 7. Timed Petri-Net where the time delay is specified by an integer value. For each transition this number is announced insight the transition.

Regarding biochemical networks time is one more important parameter. The classical definition of the Petri-Net says nothing about time (firing of a transition). Therefore, we need one more specific extension of the Petri-Net theory. Regarding the literature we can see two different approaches to model the time within 
the Petri-Net. One solution is to identify each token with his specific time stamp. More practical is to include the time defining a delay parameter for each transition (see Fig. 7). That means that the transition, which is able to fire, will fire exactly after the specified delay time. However, a global clock has to be implemented for both solutions.

Regarding the features of biochemical mechanisms we can see stochastic behavior of fundamental processes. Gene control mechanisms demonstrate that the initiation of the transcription process depends on stochastic molecular processes. The DNA-Polymerase binding process depends on the promoter sequence of the so called pribnow box, which specifies the probability value of this process. Such phenomena can be simulated using stochastic Petri-Nets [21], which allow the specification of probability values announced insight a transition (see Fig. 8).

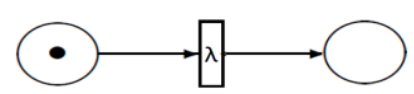

Fig. 8. This transition has concession and will fire, if a random process based on the input value lambda will produce the result true.

Regarding biochemical and molecular mechanisms we can identify positive and negative control mechanisms, like gene enhancers or transcription factors. The Hybrid Petri-Net allows modeling these effects (see Fig. 5). For Biotechnology the cell-cell communication process belongs also to the list of important phenomena, which we have to study. Modeling of cell differentiation and cell-cell communication can also be realized using Petri-Nets [24]. Therefore, we have to define the Petri-Net, which represents the biological network of the cell. The communication of such Petri-Nets can be realized by using specific transitions and places, which are able to model diffusion (see Fig. 9) or/and other communication processes.

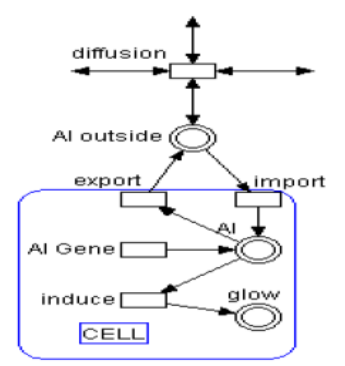

Fig. 9. The basic element is the model of a CELL. In this case the cell-cell communication is based on the diffusion process [19].

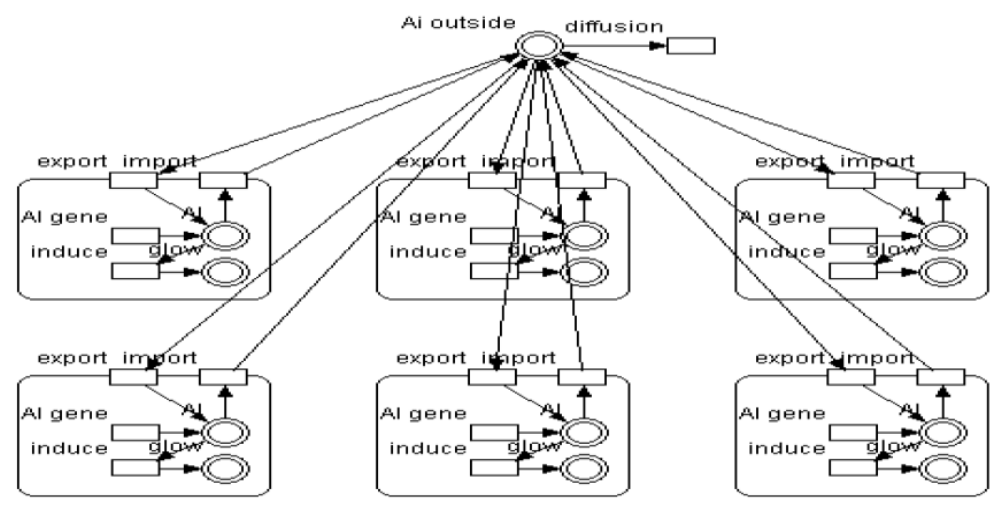

Fig. 10. The star model of cell-cell communication based on Petri-Nets [24]. 
Based on this cell model we can discuss different models for cell-cell communication. Fig. 10 is presenting the star model.

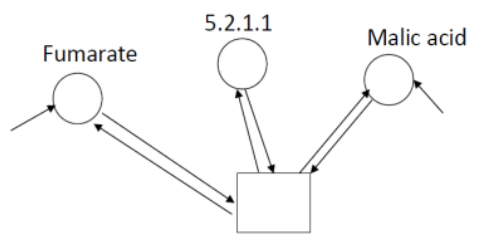

Fig. 11. Automatic Petri-Net translation of KEGG entries.

\subsection{Integrative and Automatic Generation of Petri-Nets}

The classical definition of an information system is given by a database system including data analysis tools. Based on the infrastructure of the internet this definition is more general today. Therefore, an information system is still representing a database system and a set of analysis tools. Furthermore, the internet allows access to external databases or allows the fusion of external data into the local database. During the nineties the internet came up and presented more and more molecular databases/analysis tools. Today nearly 2000 molecular databases are available via the internet [25]. Based on this situation the data fusion process helps to integrate different methods and data sources. At the beginning the so called federated database concept was common [25]. The idea of this concept was to connect the project relevant databases to the fusion software via specific adapter systems. One disadvantage of this method was/is that updates of connected database systems give rise to update the adapter system of the federated tool. One more disadvantage of this method was/is data security. To overcome these problems the warehouse concept was developed [25]. The idea of a warehouse is to integrate the user relevant external data into the new local database system using specific adapters. By this process a new database will be the result, which represent own data and integrated external data. Furthermore, tools allowing the analysis of the warehouse data are included. Based on this data integration concept different Bio-warehouse systems were developed. DAWIS-M.D. [26] is such a system based on the data warehouse infrastructure BioDWH, which integrated molecular databases, such as BRENDA, KEGG, IntAct, PIR, PDB and SIDER. Depending on ongoing projects, new databases get integrated or already integrated databases are updated. For reconstructing, visualizing, and analysis of large biological networks the VANESA system was developed and implemented [26]. Via the implemented web service to DAWIS-M.D. VANESA has access to the integrated molecular databases. It also allows users to extract information of a biological component from different biological databases. VANESA is able to edit, reconstruct, merge, intersect, combine and visualize biological networks. BioDWH, DAWIS-M.D. as well as VANESA are open-source projects and free-of-charge for academic use. Having tools allowing to reconstruct and to extend networks it is the next step to realize an automatic translation of this data into the Petri-Net notation. For this task MOVISPP [27] was developed. Access to KEGG (www.kegg.jp/kegg) allows the extraction of biochemical reactions represented by substrate, product and enzyme. The access of such data can be directly translated into the Petri-Net notation (see Fig. 11).
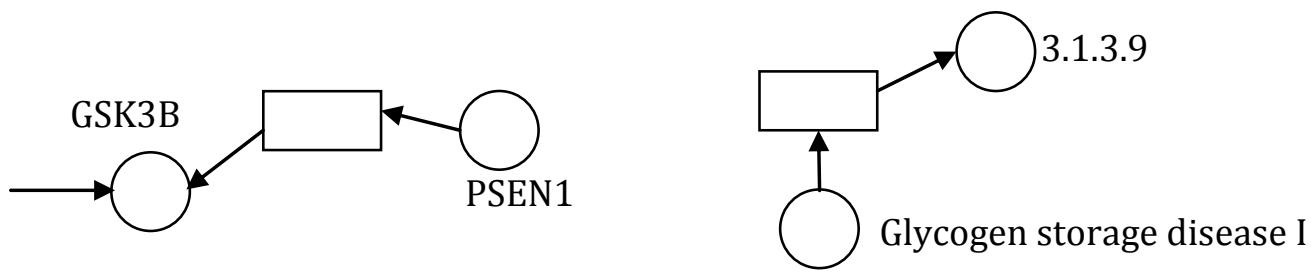

Fig. 12. Automatic Petri-Net translation of MINT (left) and OMIM (right) entries. 
MINT (http://mint.bio.uniroma2.it/) is presenting information about protein-protein interactions which can be extracted and also translated directly into the Petri-Net notation (see Fig. 12). OMIM (https://www.omim.org/) is presenting information about diseases and molecular information, which can be extracted and translated into the Petri-Net notation (see Fig. 12).

Based on such integration and fusion tools it is possible to reconstruct biological networks and to translate these networks directly into the Petri-Net notation. The MOVISPP [27] architecture is presented by Fig. 13.

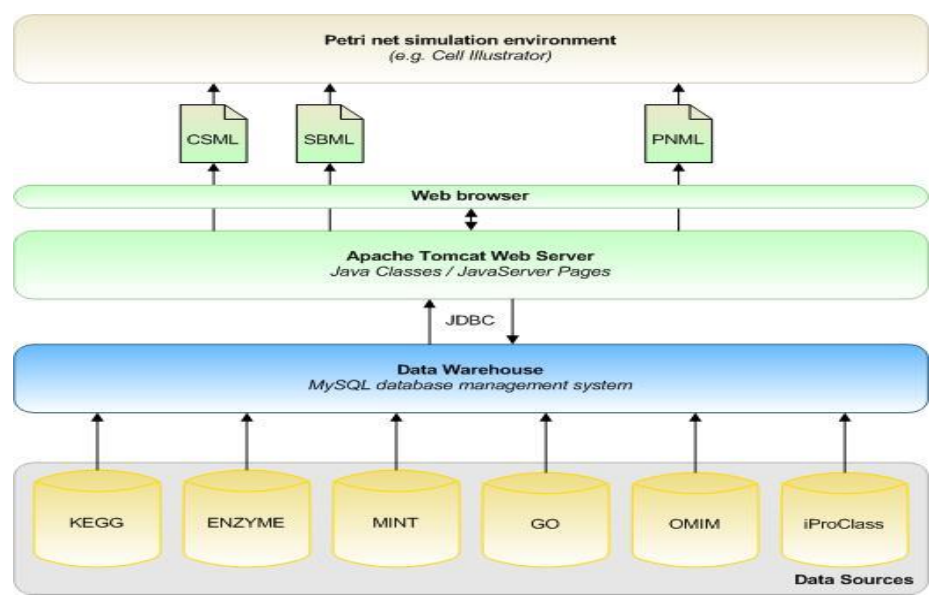

Fig. 13. Systemarchitecture of MoVisPP (modeling and visualization of pathways using Petri-Nets) [27].

\section{Generation of Petri-Net Gene Controlled Networks}

Working with VANESA [26] or MoVisPP [27] we are able to create complex biological networks based on integrated molecular data. Figure 14 is presenting a snapshot of the Bcl2/Tp53 network. The first step of this process is to identify relevant biological networks from the literature (Biochemical Pathways, Roche Applied Science) or database systems like KEGG. MoVisPP and VANESA allow the automatic access to KEGG pathways and the extension of such networks. Finally, such biological networks can be automatically translated into the Petri-Net notation. The final step is to fix the start configuration (give tokens to the places) and send the Petri-Net to the simulation tool.

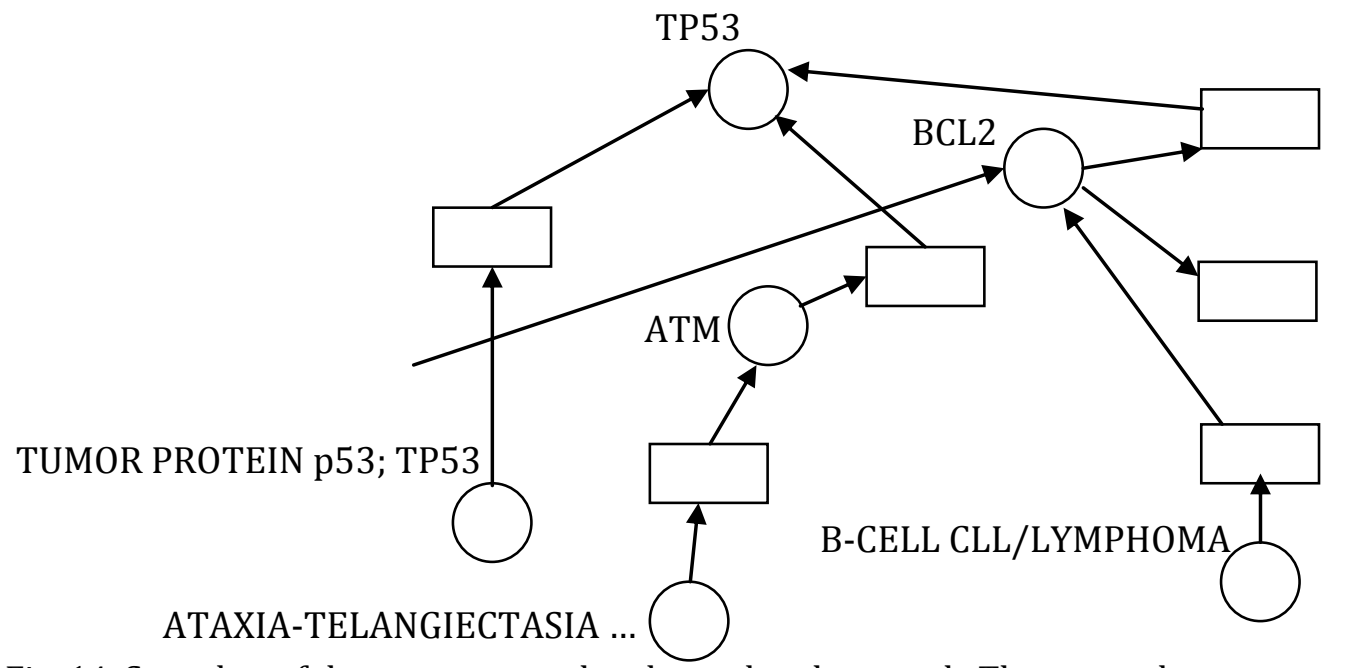

Fig. 14. Snapshot of the reconstructed and translated network. The network was extended in the area of Bcl2 and Tp53. 


\section{Conclusion}

Bioinformatics and Systems Biology both have the vision to realize the virtual cell concept. Regarding the internet we have access to the relevant knowledge of cell components today. Based on these databases and the concept of data fusion and integration we can create a virtual cell on different levels of representation. However, all biological data represents static data today. The next step is to translate this static data into a useful model and simulation environment. The idea of this paper is to announce the Petri-Net formalism as a comfortable and powerful method for the modeling and simulation of biological networks, which represent the backbone of the virtual cell project [28].

\section{References}

[1] Kauffman, S. (1969). Metabolic stability and epigenesis in randomly connected nets. Journal theor. Biol., $11,326-356$.

[2] Reddy, V. N., Mavrovouniotis, M. L., \& Liebman, M. N. (1993). Petri Net representation in metabolic pathways. Proceedings First International Conference on Intelligent Systems for Molecular Biology (pp. 328 - 336). National Library of Medicine, Bethesda.

[3] Hofestädt, R., \& Thelen, S. (1998). Quantitative modeling of biochemical networks. In Silico Biology, 1, 39-53.

[4] Mendes, P. (1993). GEPASI: A software package for modeling the dynamics, steady states and control of biochemical and other systems. Comput. Appl. Biosci., 4, 563-571.

[5] Tomita, M., Hashimoto, K., \& Takahashi, K. et al. (1999). E-CELL: Software environment for whole-cell simulation. Bioinformatics, 15, 72-84.

[6] Nagasaki, M., Saito, A., Jeong, E., Li, C., Kojima, K., Ikeda, E., \& Miyano, S. (2010). Cell-illustrator 4.0: A computational platform for systems biology. In Silico Biology, 20, 10-20.

[7] Goryanin, I., Hodgman, T., \& Selkov, E. (1999). Mathematical simulation and analysis of cellular metabolism and regulation. Bioinformatics, 15, 749-758.

[8] Loew, L. M., \& Schaff, J. C. (2001). The virtual cell: A software environment for computational cell biology. Trends Biotech, 19, 401-406.

[9] Funahashi, A., Tanimura, N., \& Morohashi, M. et al. (2003). Cell Designer: A process diagram editor for gene-regulatory and biochemical networks. BIOSILICO, 1, 159-162.

[10] Kitano, H., Funahashi, A., \& Matsuoka, Y. et al. (2005). Using process diagram for the graphical representation of biological networks. Nature Biotechnology, 23(8), 961-966.

[11] Wiechert, W. (2002). Modeling and simulation: Tools for metabolic engineering. Journal of Biotechnology, 94(1), 37-63.

[12] Rohr, C., Marwan, W., \& Heiner, M. (2010). Snoopy - A unifying Petri-Net framework to investigate biomolecular networks. Bioinformatics, 26(7), 974-975.

[13] Gronewold, A., \& Sonnenschein, M. (1998). Event-based Modeling of Ecological Systems with asynchronous cellular automata. Ecological Modeling, 108, 37-52.

[14] Proß, S., \& Bachmann, B. (2014). An object-oriented Petri net simulation tool for hybrid biological processes. It Information Technology, 56(2), 55-66.

[15] Nagasaki, M., Doi, A., \& Matsuno, H. et al. (2003) Genomic object net: A platform for modelling and simulating biopathways. Applied Bioinformatics, 2(3), 181-192.

[16] Reisig, W. A. (1982). Primer in Petri-Net Design. Springer Verlag, Berlin.

[17] Alla, H., \& David, R. (1998). Continuous and hybrid petri nets. Journal of Circuits Systems Computers, $8(1), 59-88$.

[18] Nagasaki, M., Doi, A., \& Matsuno, H. (2004). Integrating biopathway databases for large-scale modeling 
and simulation. In Second Asia-Pacific Bioinformatics Conferences, Volume 29 of Conferences in Research and Practice in Information Technology, Australian Computer Society, 43-52.

[19] Drath, R. (1998). Hybrid object nets: An object oriented concept for modeling complex hybrid systems. 3rd International Conference on Automation of Mixed Processes, 437-442.

[20] Doi, A., Fujita, S., Matsuno, H., Nagasaki, M., \& Miyano, S. (2004). Constructing biological pathway models with hybrid functional petri-nets. In Silico Biology, 4, 271-291.

[21] Balazki, P., Lindauer, K., Einloft, J., Ackermann, J., \& Koch, I. (2015). MONALISA for stochastic simulations of Petri-Net models of biochemical systems. BMC Bioinformatics, 16, 371.

[22] Liu, F., Heiner, M., \& Yang, M. (2016). Fuzzy stochastic petri nets for modeling biological systems with uncertain kinetic parameters. PLOS ONE, 11(2), 33-45.

[23] Liu, C., You, J., You, X., \& Su, Q. (2016). Linguistic reasoning: Petri nets for knowledge representation and reasoning. IEEE Trans. Syst. Man Cybern. Syst., 46(4), 499-511.

[24] Janowski, S., Kormeier, B., Töpel, T., Hippe, K., Hofestädt, R., Willassen, N., Friesen, R., Rubert, S., Borck, D., Haugen, P., \& Chen, M. (2011). Modeling of cell-to-cell communication processes with Petri-Nets using the example of quorum sensing. Stud Health Technol. Inform., 162, 33-49.

[25] Chen, M., \& Hofestädt, R. (2014). Approaches in integrative bioinformatics - Towards the virtual cell. Springer Verlag, Heidelberg.

[26] Brinkrolf, C., Janowski, S., Kormeier, B., Lewinski, M., Hippe, K., Borck, D., \& Hofestädt, R. (2014). VANESA - A software application for the visualization and analysis of networks in systems biology applications. Journal of Integrative Bioinformatics, 11(2), 54-62.

[27] Chen, M., Hariharaputran, S., Hofestädt, R., Kormeier, B., \& Spangardt S. (2011). Petri-Net models for the semi-automatic construction of large scale biological networks. Natural Computing, 10(3), 1077-1097.

[28] Chen, M., \& Hofestädt, R. (2014). Open problems in Petri-Net modeling and simulation of biological systems. It Information Technology, 56(2), 76-81.

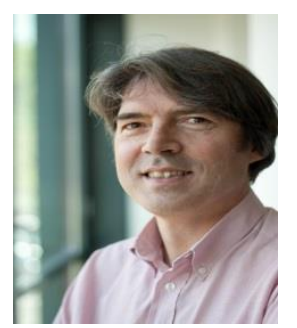

Ralf Hofestädt studied computer science and bioinformatics at the University of Bonn. He finished his PhD 1990 (University Bonn) and his habilitation for applied computer science and bioinformatics 1995 (University of Koblenz). From 1996 to 2001 he was professor for applied computer science at the University of Magdeburg. Since 2001 he is professor for bioinformatics and medical informatics at Bielefeld University. 